COMMENT

\title{
Fasting-mimicking diet plus chemotherapy in breast cancer treatment
}

\author{
Claudio Vernieri (10 ${ }^{1,2}$, Francesca Ligorio ${ }^{1}$, Emma Zattarin ${ }^{1}$, Licia Rivoltini ${ }^{3} \&$ \\ Filippo de Braud ${ }^{1,4}$
}

\begin{abstract}
A clinical trial published in Nature Communications examined the effect of fastingmimicking diet (FMD) during chemotherapy in breast cancer patients. The overall negative study results highlight the need for ameliorating future trial design and investigating alternative FMD-based therapeutic combinations.
\end{abstract}

\section{The "DIRECT" trial}

In a study recently published in Nature Communications, de Groot et al. reported the results of the phase II trial "DIRECT", in which experimental cyclic fasting-mimicking diet (FMD) in combination with standard anthracycline-taxane preoperative chemotherapy (ChT) failed to reduce ChT-related adverse events and to improve the rate of pathological complete responses (pCR) in patients with stage II-III HER2-negative breast cancer (BC) ${ }^{1}$. However, some study findings, such as the possibility to avoid dexamethasone use during doxorubicincyclophosphamide ChT and the reduction of ChT-induced DNA damage to circulating lymphocytes in patients undergoing the FMD, are of potential interest. While highlighting the importance of publishing results of clinical trials even when they are negative for their primary endpoint, findings of the DIRECT trial indicate the necessity to ameliorate patient adherence to the FMD and to improve clinical trial designs to fully exploit the therapeutic potentialities of calorie-restricted dietary interventions.

In tumor-bearing mice, cycles of fasting or calorie-restricted, low-carbohydrate, low-protein diets, collectively referred to as FMDs, synergize with cytotoxic ChT or other antitumor therapies to slow down tumor growth ${ }^{2,3}$. At the same time, fasting/FMD protect normal tissues from ChTinduced toxicity ${ }^{4,5}$ and boosts ChT-induced CD8 $+\mathrm{T}$ cell intratumor infiltration ${ }^{2}$. These effects are mainly mediated by fasting/FMD-induced reduction of blood glucose, insulin and insulinlike growth factor 1 (IGF-1) concentration ${ }^{4,5}$. Notably, murine models of BC are exquisitely sensitive to the FMD when compared to other tumor types ${ }^{2,3}$. Therefore, the DIRECT trial, which investigated the FMD efficacy in reducing ChT-induced toxicities and in increasing pCR rates in HER2- BC patients, was timely and based on solid preclinical evidence. In addition, the study was conducted in early-stage BC patients, who are at low risk of undergoing malnutrition and cachexia,. Finally, this was a randomized phase II/III trial with sufficient power to provide evidence of a clinical benefit of the FMD in a selected population of cancer patients. For these

\footnotetext{
${ }^{1}$ Medical Oncology and Hematology Department, Fondazione IRCCS Istituto Nazionale dei Tumori, Via Venezian 1, 20133 Milan, Italy. ${ }^{2}$ Metabolic Reprogramming in Solid Tumors Unit. IFOM, the FIRC Institute of Molecular Oncology, Via Adamello 16, 20139 Milan, Italy. ${ }^{3}$ Unit of Immunotherapy of Human Tumors, Fondazione IRCCS Istituto Nazionale dei Tumori, Via Venezian 1, 20133 Milan, Italy. ${ }^{4}$ Oncology and Hemato-Oncology Department, University of Milan, 20122 Milan, Italy. ${ }_{\text {email: claudio.vernieri@ifom.eu }}$
} 
reasons, the DIRECT trial had the potential to provide proof-ofconcept demonstration that the FMD positively impacts on ChT tolerability and antitumor activity in BC patients.

Unfortunately, the study was prematurely interrupted because a pre-planned interim analysis showed a lower-than-anticipated pCR rate in the study population, as well as poor patient adherence to the proposed FMD regimen ${ }^{1}$. In addition, the FMD did not provide evident clinical advantages, neither in terms of reduction of AEs (the primary endpoint of the phase II trial) nor in terms of increased pCR rates (the primary endpoint of the phase III trial) ${ }^{1}$. Of note, triple-negative breast cancer (TNBC) patients, who are much more likely to undergo pCR during preoperative $\mathrm{ChT}^{6}$, were significantly more represented in the FMD arm than in the control arm (21.5\% vs. $10.9 \%)$. Therefore, an increase of pCR rates in the experimental arm would have been expected also independently of the FMD. Although the authors emphasized the occurrence of higher rates of clinical/ radiological tumor responses in patients receiving the ChT-FMD combination, the clinical relevance of this finding is questionable. Indeed, while pCR is a suitable surrogate biomarker of preoperative ChT efficacy and a predictor of long-term clinical outcomes $^{6}$, radiological responses are only poorly-to-moderately associated with $\mathrm{pCR}^{7}$, and their impact on long-term outcomes is uncertain.

\section{Improving patient adherence to the FMD: a crucial issue}

One reason that could explain the negative results of the DIRECT trial is low patient compliance with the experimental FMD regimen. Indeed, although the maximum number of allowed FMD cycles was 8 as per protocol, only $\sim 50 \%$ and $33.8 \%$ of patients were able to complete at least 2 and 4 FMD cycles, respectively. Such low compliance rates might have crucially contributed to the failure to reduce ChT-induced adverse events and to increase pCR rates in the experimental arm.

In mouse models, two fasting/FMD cycles in combination with ChT can be sufficient to slow-down tumor growth in the shortterm period ${ }^{2}$, but a higher number of FMD cycles is required to achieve long-term tumor control. In the case of human cancers, which are characterized by higher biological complexity and heterogeneity when compared to smaller murine neoplasms ${ }^{8}$, completing a higher number of FMD cycles might be even more important to observe some clinical benefit. Consistent with the concept that patient adherence to the FMD is a crucial prerequisite for its antitumor activity, a per-protocol sub-analysis of the DIRECT trial showed that compliant patients had significantly higher rates of pathological tumor responses according to the Miller-Payne $4 / 5$ scores (which may approximate pCR as a predictor of tumor recurrences limited to TNBC subgroup ${ }^{9}$ ), and also reported lower DNA damage to peripheral blood lymphocytes.

The main cause of lack of adherence to the experimental diet was dislike of specific components of the FMD, which consisted of a plant-based, standardized kit providing $\sim 1200 \mathrm{kcal}$ on day 1 and $\sim 200 \mathrm{kcal}$ on days $2-4^{1}$. Alternative FMD regimens, including schemes containing fresh foods, could be associated with better patient compliance, thus potentially resulting in higher antitumor activity ${ }^{10}$. Four clinical trials are currently being conducted at our institution to investigate the safety, feasibility, metabolic, immunological, and antitumor activity of cyclic FMD in combination with standard therapies in patients with different tumor types (ClinicalTrials.gov Identifiers: NCT03340935; NCT03454282; NCT03709147; NCT04248998). The FMD scheme used in these studies, which provides $\sim 600 \mathrm{kcal}$ on day 1 and $\sim 300 \mathrm{kcal}$ on days $2-5$, consists of a list of permitted fresh foods and beverages, which patients can choose according to their preferences. Results of ongoing studies (Table 1) will clarify if more flexible and physiological FMD schemes can guarantee better patient compliance, thus becoming the reference FMD regimen to be tested in future clinical trials.

In parallel with poor compliance with experimental dietary regimens, another limitation of clinical trials investigating dietary interventions consists in the fact that patients randomized to the control diet can spontaneously reduce their calorie intake, thus confounding the study findings. In the DIRECT trial, only $7.8 \%$ of patients in the control arm admitted that they decided to fast, thus making it unlikely that their deviations impacted on the study findings. However, due to the relevance of this issue, alternative study designs, including cross-over trials, or randomized trials employing placebo diets with similar appearance to FMD kits, but with calorie and macronutrient content that is consistent with dietary recommendations for cancer patients (https://www.wcrf. org/dietandcancer/recommendations-about $)^{11}$, should be considered in future trials.

\section{FMD to spare dexamethasone use and boost antitumor immunity}

One interesting finding of the DIRECT trial is that patients undergoing the FMD did not receive dexamethasone as a premedication to doxorubicin-cyclophosphamide (AC) ChT, and yet they did not experience a higher incidence of AEs when compared to patients in the control arm. These data indirectly suggest that the FMD could protect patients from some ChT-induced AEs (e.g. nausea, vomiting), thus making dexamethasone premedication unnecessary. Since dexamethasone could promote BC metastasis $^{12}$ and result in potentially detrimental metabolic and immunological effects ${ }^{13}$, avoiding dexamethasone administration could per se enhance ChT-induced anticancer effects in specific clinical contexts. Although intriguing, this hypothesis needs to be tested in future prospective trials.

Preclinical in vivo studies showed that two fasting/FMD cycles boost antitumor immunity and promote intratumor infiltration by $\mathrm{CD}^{+} \mathrm{T}$ cells through reducing blood IGF-1 levels, which is an essential determinant of the anticancer effects of calorie restriction ${ }^{2}$. If this also applies to human cancers, 1-2 FMD cycles, which were completed by the majority of patients in the DIRECT trial, might be sufficient to activate antitumor immunity. In this perspective, the observed reduction of DNA damage to circulating lymphocytes in compliant patients is interesting. If the FMD boosts antitumor immunity in cancer patients similarly to tumor-bearing mice, FMD-induced clinical benefit might become apparent in the long-term period, thus resulting in reduced tumor recurrences and improved long-term outcomes despite the lack of pCR advantages. In this perspective, it will be interesting to analyze relapse-free survival and overall survival (OS) data from the DIRECT study when they are mature.

\section{How to improve FMD antitumor efficacy in future trials?}

FMD antitumor activity could be attenuated by adaptive metabolic mechanisms allowing cancer cells to survive also in conditions of glucose and growth factor deprivation. For instance, cancer cells can adapt to fasting/FMD-induced reduction of extracellular glucose by increasing the utilization of mitochondrial oxidative phosphorylation (OXPHOS) as an alternative source of energy units (ATP) and anabolic precursors. One recent study showed that the simultaneous inhibition of tumor glycolysis (through cycling fasting) and OXPHOS (through metformin) produces synergistic and long-lasting anticancer effects in tumor cell lines and tumor-bearing mice, which are mediated through a potent activation of the PP2A-GSK3 $\beta$ tumor suppressor axis ${ }^{14}$. 


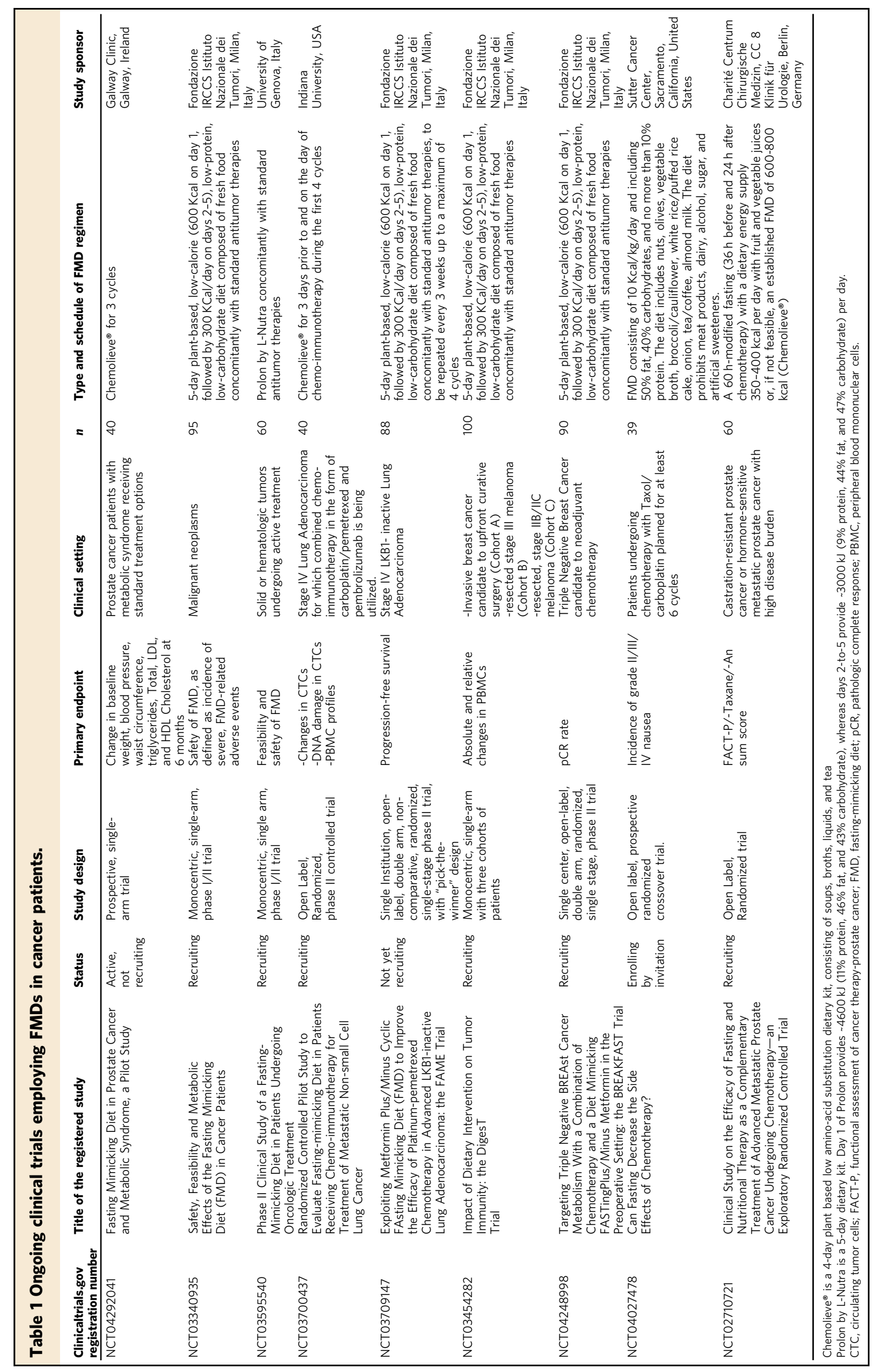


Based on these data, we have recently initiated the phase II, randomized study "BREAKFAST" (NCT04248998) to test the anticancer activity of adding cyclic FMD, or FMD plus metformin, to standard preoperative ChT in stage I-III TNBC patients (Table 1).

Received: 27 June 2020; Accepted: 12 August 2020;

Published online: 26 August 2020

\section{References}

1. de Groot, S. et al. Fasting mimicking diet as an adjunct to neoadjuvant chemotherapy for breast cancer in the multicentre randomized phase 2 DIRECT trial. Nat. Commun. 11, 3083 (2020).

2. Di Biase, S. et al. Fasting-mimicking diet reduces HO-1 to promote T cellmediated tumor cytotoxicity. Cancer Cell 30, 136-146 (2016).

3. Caffa, I. et al. Fasting-mimicking diet and hormone therapy induce breast cancer regression. Nature 583, 620-624 (2020).

4. Brandhorst, S. et al. A periodic diet that mimics fasting promotes multi-system regeneration, enhanced cognitive performance, and healthspan. Cell Metab. 22, 86-99 (2015).

5. Raffaghello, L. et al. Starvation-dependent differential stress resistance protects normal but not cancer cells against high-dose chemotherapy. Proc. Natl Acad. Sci. USA 105, 8215-8220 (2008).

6. Cortazar, P. et al. Pathological complete response and long-term clinical benefit in breast cancer: the CTNeoBC pooled analysis. Lancet 384, 164-172 (2014).

7. Romero, A. et al. Correlation between response to neoadjuvant chemotherapy and survival in locally advanced breast cancer patients. Ann. Oncol. 24, 655-661 (2013).

8. Guerin, M. V., et al. Preclinical murine tumor models: a structural and functional perspective. Elife 9, https://doi.org/10.7554/eLife.50740 (2020).

9. Choi, M. et al. Assessment of pathologic response and long-term outcome in locally advanced breast cancers after neoadjuvant chemotherapy: comparison of pathologic classification systems. Breast Cancer Res Treat. 160, 475-489 (2016).

10. Bauersfeld, S. P. et al. The effects of short-term fasting on quality of life and tolerance to chemotherapy in patients with breast and ovarian cancer: a randomized cross-over pilot study. BMC Cancer 18, 476 (2018).

11. Cheryl L. et al. American Cancer Society guideline for diet and physical activity for cancer prevention ACS journal. https://doi.org/10.3322/caac.21591 (2020).

12. Obradovic, M. M. S. et al. Glucocorticoids promote breast cancer metastasis. Nature 567, 540-544 (2019).
13. Giles, A. J. et al. Dexamethasone-induced immunosuppression: mechanisms and implications for immunotherapy. J. Immunother. Cancer 6, 51 (2018).

14. Elgendy, M. et al. Combination of hypoglycemia and metformin impairs tumor metabolic plasticity and growth by modulating the PP2A-GSK3betaMCL-1 Axis. Cancer Cell 35, 798-815 e795 (2019).

\section{Acknowledgements}

We would like to thank the "Associazione Italiana per la Ricerca sul Cancro" (AIRC) for funding our research (IG-2017 n.20752: PI Licia Rivoltini; MFAG-2019 n. 22977: PI Claudio Vernieri).

\section{Author contributions}

C.V. and F.deB conceived this work. C.V., F.L. and E.Z. wrote the manuscript, with critical revision by L.R. and F.deB. All the authors read and approved the final version of the manuscript.

\section{Competing interests}

C.V., L.R. and F.deB. were inventors of an FMD regimen that is being evaluated in the context of ongoing clinical trials in cancer patients.

\section{Additional information}

Correspondence and requests for materials should be addressed to C.V.

Reprints and permission information is available at http://www.nature.com/reprints

Publisher's note Springer Nature remains neutral with regard to jurisdictional claims in published maps and institutional affiliations.

\begin{abstract}
Open Access This article is licensed under a Creative Commons Attribution 4.0 International License, which permits use, sharing, adaptation, distribution and reproduction in any medium or format, as long as you give appropriate credit to the original author(s) and the source, provide a link to the Creative Commons license, and indicate if changes were made. The images or other third party material in this article are included in the article's Creative Commons license, unless indicated otherwise in a credit line to the material. If material is not included in the article's Creative Commons license and your intended use is not permitted by statutory regulation or exceeds the permitted use, you will need to obtain permission directly from the copyright holder. To view a copy of this license, visit http://creativecommons.org/ licenses/by/4.0/.
\end{abstract}

(C) The Author(s) 2020 